\title{
Cirurgia paliativa do ombro em paralisia obstétrica do plexo braquial
}

\author{
C. Santos ${ }^{(1)}$, A. Pereira ${ }^{(1)}$, R. Rodrigues Pinto ${ }^{(1)}$, M. Trigueiros $^{(2)}$, \\ R. $\operatorname{LEMOS}^{(3)}$, C. SILVA ${ }^{(3)}$ \\ Serviço de Ortopedia. Hospital de Santo António - Centro Hospitalar do Porto. Portugal \\ (1) INTERNO COMPLEMENTAR \\ (2) ASSISTENTE HoSPITALAR \\ (3) ASSISTENTE GRADUADO
}

Correspondência:

Dra. Cláudia Santos

Serviço de Ortopedia. Hospital Santo António

Largo Professor Abel Salazar

4099-001 Porto

Telef. 222077500 - Fax. 222087632

e-mail: claudsantos@hotmail.com

A paralisia obstétrica do plexo braquial é rara originando disfunção grave e défice marcado da rotação externa e da abdução do ombro. Reviram-se retrospectivamente 11 casos de paralisia obstétrica do plexo braquial (sete com paralisia alta e quatro com paralisia completa) tratados cirurgicamente pela técnica de L'Épiscopo modificada por Hoffer. Estes resultados foram padronizados segundo o tipo de lesão (grupo A - lesão alta; grupo B - lesão completa) e a idade (grupo I $\leq 4$ anos; grupo II $>4$ anos). O seguimento médio foi de 35 meses. A idade média aquando da cirurgia era de 6,5 anos $(\mathrm{r}=2-11)$. Todos, excepto um, apresentaram melhoria funcional, com aumento da pontuação média de Mallet de 4,6 pontos. Os dois subtipos de paralisia tiveram pontuação média de Mallet sobreponível (grupo A - 4.6; grupo B - 4.7); a idade aquando da cirurgia não influenciou o resultado clínico (pontuação média (grupo I) - 4.8; (grupo II) - 4.4). Não foram registadas complicações. A técnica de L'Épiscopo modificada por Hoffer é eficaz no tratamento paliativo do ombro em paralisia obstétrica do plexo braquial, permitindo recuperação da abdução e rotação externa do ombro. Está indicada em paralisias altas e completas, quando associada a outras cirurgias de recuperação da função do cotovelo, punho e/ou mão. Idade superior a 4 anos não contra-indica a cirurgia
The obstetric brachial plexus palsy is a rare disorder causing severe deficit and limited external rotation and abduction. 11 cases of obstetric brachial plexus palsy were retrospectively reviewed (seven with high paralysis and four with complete paralysis) and surgically treated by the technique of L'episcope modified by Hoffer. These results were stratified according to the type of lesion (group A - high injury, group B - complete injury) and age (group $\mathrm{I} \leq 4$ years, group II $>4$ years). The mean follow-up was 35 months. The mean age at the time of surgery was 6.5 years $(r=2-11)$. All but one showed functional improvement, with increased mean Mallet score of 4.6 points. The two subtypes of paralysis had similar mean Mallet score (group A - 4.6; group B - 4.7); the age at the time of surgery did not influence the clinical outcome (average score (Group I) - 4.8, (Group II) - 4.4). No complications were recorded. The technique L'episcope modified by Hoffer is effective in the palliative treatment of the shoulder in obstetric brachial plexus palsy, allowing recovery of abduction and external rotation of the shoulder. It seems to be indicated for high and complete paralysis, when combined with other surgeries to recover the function of the elbow, wrist and / or hand. Being older than 4 years old is not a contraindication for 
desde que sejam excluídas alterações da articulação glenoumeral.

Palavras chave: paralisia obstétrica do plexo braquial, operação de l'épiscopo modificada, escala funcional de Mallet modificada this surgery provided that changes of the glenohumeral joint are excluded.

Key words: obstetric brachial plexus palsy, l'episcope modified operation; modified Mallet scale

\section{INTRODUÇÃO}

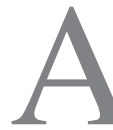
incidência da Paralisia Obstétrica do Plexo Braquial (POPB) está a diminuir como resultado de melhores cuidados perinatais e do aumento da prática de cesariana. Contudo ainda ocorre numa taxa de 0,1 a $0,4 \%{ }^{1,2}$. Aproximadamente $85 \%$ dos casos diagnosticados após o parto recuperam espontaneamente $^{3}$. As restantes POPB manifestam-se geralmente como paralisia alta do plexo ou tipo de Erb (C5, C6 e por alguns autores C7); com défice essencialmente na abdução e rotação externa do ombro ou, mais raramente, como paralisia completa (C5 a T1), com défices musculares mais distais do membro superior afectado.

A persistência destes desequilíbrios musculares leva a contractura do membro superior em adução e rotação interna e, consequentemente, a alterações articulares da glenoumeral com instabilidade posterior do ombro, que pode ser irreversível se não tratada precocemente ${ }^{4-6}$.

Desde o início do século XX, têm sido descritas várias cirurgias para o tratamento da POPB, desde técnicas de reconstrução microcirúrgica do plexo braquial $^{7}$ a procedimentos paliativos. Em 1913, Fairbank defende a libertação do subescapular e capsulotomia anterior ${ }^{8}$; mais tarde em 1925, Sever descreve a libertação do subescapular e grande peitoral ${ }^{6}$. L'Épiscopo em 1934 publica pela primeira vez a técnica que permite, não só corrigir a contractura em adução e rotação interna, mas também recuperar a abdução e rotação externa. Para além da tenotomia do subescapular e grande peitoral, descreve a transferência do grande dorsal e grande redondo da face anteromedial para posteromedial do úmero proximal $^{9}$. Em 1978, Hoffer et al. modificam a técnica de L'Épiscopo transferindo o grande dorsal e grande redondo para a coifa dos rotadores ${ }^{10}$. Mais recentemente, em 2006, Pearl e al. defendem a técnica artroscópica para libertação do subescapular e capsulotomia ${ }^{11}$.

\section{MATERIAL E MÉTODO}

Durante o período de 2000 a 2008 foram submetidos a cirurgia paliativa do ombro 11 doentes com POPB (Tabela I). A técnica cirúrgica utilizada foi semelhante à descrita por Hoffer et al. ${ }^{10}$ : libertação do subescapular e grande peitoral e transferência do grande dorsal e grande redondo para a coifa dos rotadores (Figura 1 e 2).

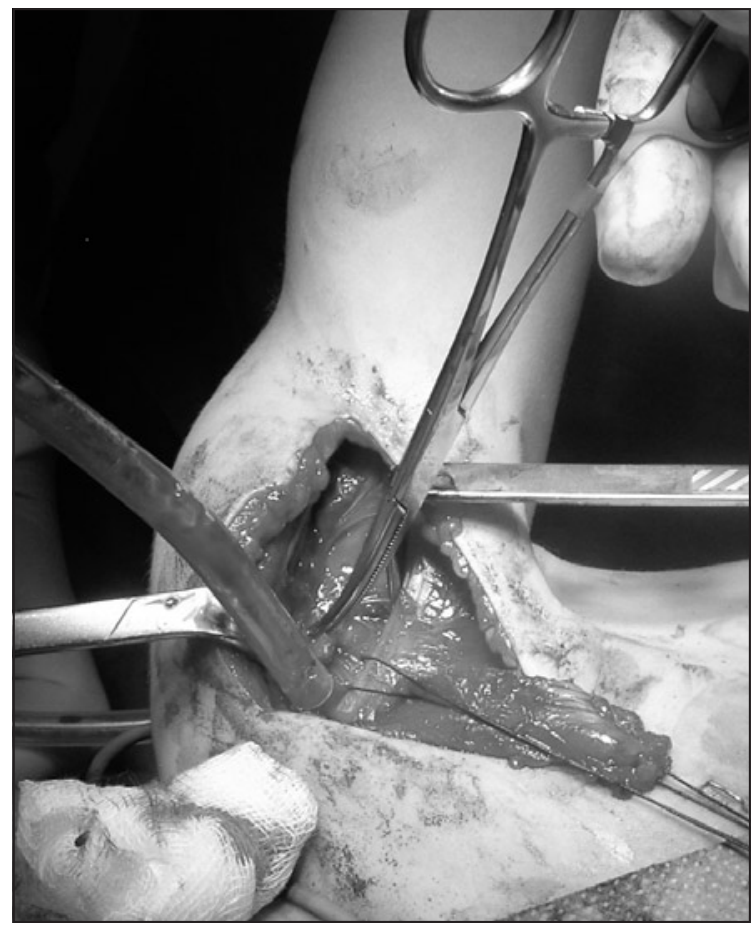

Figura 1: Abordagem anterior com tenotomia do grande peitoral e subescapular para alongamento. 


\section{TABela I - Dados dos doentes}

\begin{tabular}{|c|c|c|c|c|c|c|c|}
\hline Caso & $\begin{array}{c}\text { Sexo } \\
\text { (M/F) }\end{array}$ & $\begin{array}{c}\text { Idade } \\
\text { aquando } \\
\text { da cirurgia } \\
\text { (anos) }\end{array}$ & $\begin{array}{c}\text { Data da } \\
\text { cirurgia }\end{array}$ & Tipo & $\begin{array}{c}\text { Direito (D)/ } \\
\text { Esquerdo } \\
\text { (E) }\end{array}$ & $\begin{array}{c}\text { Seguimento } \\
\text { (meses) }\end{array}$ & Outras cirurgias \\
\hline 1 & $\mathrm{~F}$ & 9 & $7 / 2006$ & Alta & E & 24 & Não \\
\hline 2 & $\mathrm{~F}$ & 3 & $2 / 2005$ & Completa & $\mathrm{D}$ & 42 & Exploração do plexo aos 13 meses \\
\hline 3 & $\mathrm{M}$ & 2 & $12 / 2005$ & Completa & $\mathrm{D}$ & 32 & $\begin{array}{c}\text { Transferência pequeno peitoral para } \\
\text { bíceps 5 anos }\end{array}$ \\
\hline 4 & $\mathrm{M}$ & 3 & $9 / 2005$ & Alta & $\mathrm{D}$ & 34 & Não \\
\hline 5 & $\mathrm{M}$ & 5 & $11 / 2007$ & Alta & $\mathrm{E}$ & 20 & Não \\
\hline 6 & $\mathrm{~F}$ & 7 & $6 / 2006$ & Alta & $\mathrm{D}$ & 24 & $\begin{array}{c}\text { Exploração do plexo 5 meses } \\
\text { Transferência triceps 3 anos }\end{array}$ \\
\hline 7 & $\mathrm{~F}$ & 2 & $2 / 2003$ & Completa & $\mathrm{E}$ & 66 & $\begin{array}{c}\text { Transferência extensor radial longo } \\
\text { do carpo para interósseo de wM3 e } \\
\text { M4 aos 6 anos }\end{array}$ \\
\hline 8 & $\mathrm{M}$ & 3 & $9 / 2005$ & Alta & D & 34 & Não \\
\hline 9 & $\mathrm{~F}$ & 2 & $4 / 2000$ & Completa & D & 96 & $\begin{array}{c}\text { Exploração do plexo aos 3 meses } \\
\text { Operação de Steindler aos 3 anos } \\
\text { Transferência de FCC para ECRB }\end{array}$ \\
\hline 10 & $\mathrm{~F}$ & 6 & $6 / 2007$ & Alta & $\mathrm{D}$ & 13 & Não \\
\hline 11 & $\mathrm{~F}$ & 11 & $6 / 2008$ & Alta & E & 3 & Não \\
\hline
\end{tabular}

A idade média na data da cirurgia foi de 6,5 $\operatorname{anos}(r=2-11)$. Sete são do sexo feminino e 4 do sexo masculino. Quatro são do membro superior direito e 7 do esquerdo. Sete doentes têm paralisia alta e 4 paralisia completa, isto

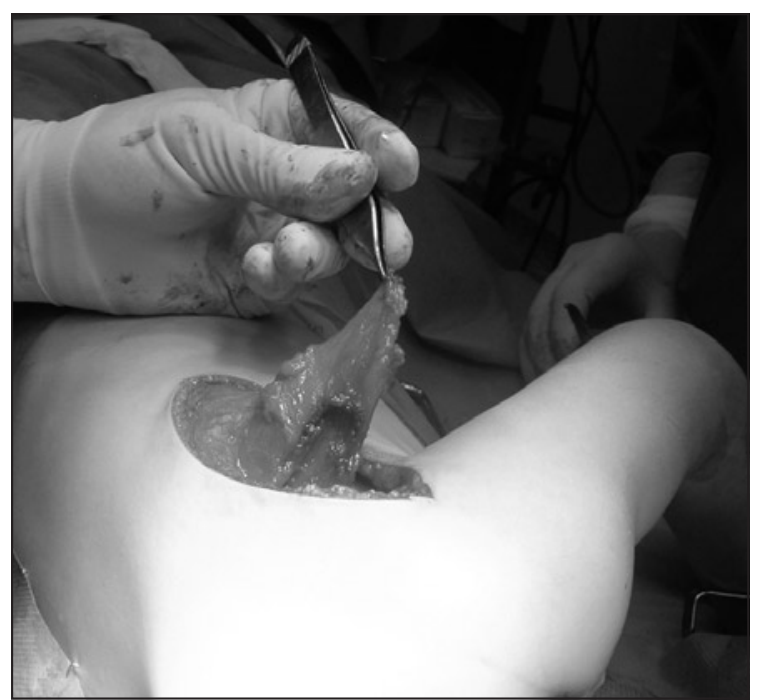

Figura 2: Abordagem posterior com tenotomia do grande dorsal e grande redondo para transferência para a coifa dos rotadores. é, paralisia total à nascença com recuperação parcial da alguns grupos musculares, espontaneamente ou após cirurgia de exploração do plexo braquial. Foram excluídas crianças com diminuição da força muscular do grande dorsal e grande redondo. Todos os doentes foram estudados imagiologicamente com RX e alguns com TAC, não tendo sido detectadas importantes alterações da articulação glenoumeral. Os doentes com paralisias completas foram submetidos a outras cirurgias para correcção da função do cotovelo, punho e/ou mão. O protocolo pós-operatório consistiu em imobilização gessada toracobraquial com o membro superior em abdução e rotação externa durante 5 a 6 semanas (Figura 3), após as quais são iniciados exercícios de fisioterapia. O seguimento médio foi de 35 meses $(r=3-96)$.

Para avaliar a função do ombro foi utilizada a escala de Mallet modificada ${ }^{12}$ no pré e pósoperatório. Em Junho de 2008 foram reavaliados todos os doentes e calculada a pontuação final de Mallet (Tabela II). No grupo das paralisias completas esta avaliação é o somatório das diferentes cirurgias efectuadas ao membro superior. 


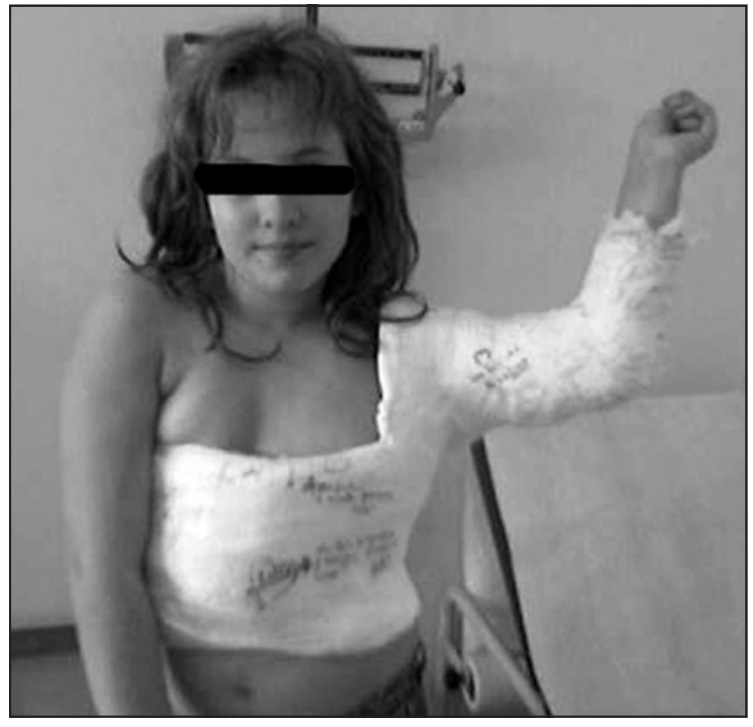

Figura 3: Imobilização gessada toracobraquial.

Para comparar os resultados quanto ao tipo de lesão, dividiram-se os doentes em grupos, quanto ao tipo de lesão em grupo A (lesão alta) e grupo B (lesão completa) e quanto à idade na data de cirurgia, em grupo I (idade $\leq 4$ anos) e grupo II (idade $>4$ anos). Considerou-se como «aumento da pontuação» a diferença entre a pontuação de Mallet no pós-operatório e préoperatório. A «pontuação média» do grupo é a razão entre o aumento da pontuação e o total dos doentes desse grupo.

\section{RESULTADOS}

A avaliação funcional de Mallet no pré-operatório variou de 6 a 10 valores e no pós-operatório de 8 a 15 valores. Verificou-se um aumento da pontuação de 0 a 8 valores, com média de 4.6 (Tabela III).

Quanto ao tipo de lesão (Tabela IV), o grupo A variou de 8 a 10 valores na escala de Mallet no pré-operatório e de 12 a 15 no pós-operatório. $\mathrm{O}$ aumento médio da pontuação foi de 4,6. No grupo $\mathrm{B}$, variou de 5 a 8 valores no pré e 8 a 14 no pós-operatório, com um aumento da pontuação média de 4,7 .

No que diz respeito à idade na data da cirurgia (Tabela V), o grupo I variou de 0 a 6 valores, com pontuação média de 4,8 e o grupo II de 3 a 6 valores, com pontuação média de 4.4 .
Não foram registadas quaisquer complicações, nomeadamente perdas sanguíneas significativas com necessidade de transfusão, lesões neurovasculares, infecções, cicatrizes retrácteis ou úlceras de pressão causadas pela imobilização gessada prolongada.

\section{DISCUSSÃO}

Verificou-se um aumento da pontuação na avaliação de Mallet em todos os doentes excepto 1 (caso 3). Estes resultados explicam-se pela eficácia da técnica de L'Épiscopo modificada, tal como defendida por Hoffer et al. ${ }^{10}$. Não encontrámos nenhuma causa concreta que justifique o único caso de insucesso que tivemos. Pode ter ocorrido uma deiscência de sutura da anastomose tendinosa, lesão de nervo motor, tensão insuficiente da sutura ou uma incorrecta avaliação da força muscular pré-operatória.
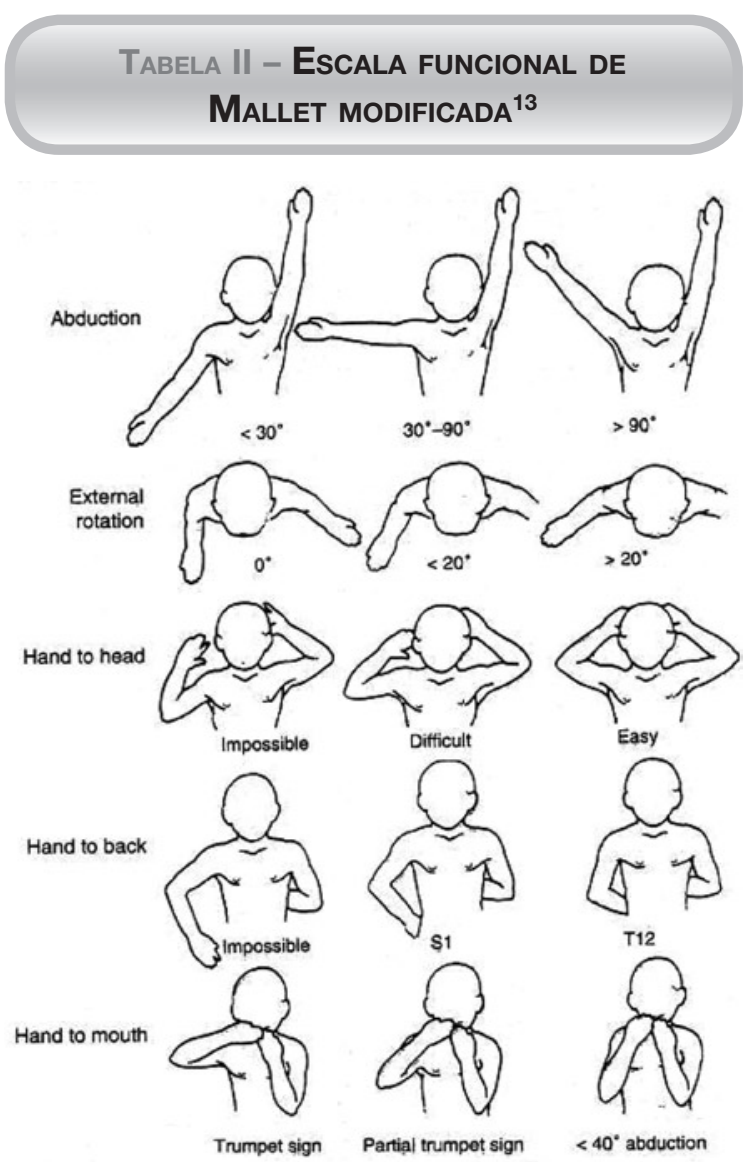

Value

II

III

IV 


\section{Tabela III - Resultados globals}

\begin{tabular}{|c|c|c|c|}
\hline Caso & $\begin{array}{c}\text { Mallet pré- } \\
\text { operatório }\end{array}$ & $\begin{array}{c}\text { Mallet pós- } \\
\text { operatório }\end{array}$ & $\begin{array}{c}\text { Aumento } \\
\text { pontuação }\end{array}$ \\
\hline 1 & 10 & 13 & 3 \\
\hline 2 & 6 & 14 & 8 \\
\hline 3 & 8 & 8 & 0 \\
\hline 4 & 9 & 14 & 5 \\
\hline 5 & 9 & 12 & 3 \\
\hline 6 & 8 & 13 & 5 \\
\hline 7 & 5 & 11 & 6 \\
\hline 8 & 8 & 13 & 5 \\
\hline 9 & 8 & 13 & 5 \\
\hline 10 & 10 & 15 & 5 \\
\hline 11 & 8 & 14 & 6 \\
\hline & \multicolumn{3}{|r}{} \\
\hline
\end{tabular}

\section{TABela IV - Resultados SEgundo o TIPO DE PARALISIA}

Grupo A: Paralisia alta

\begin{tabular}{|c|c|c|c|}
\hline Caso & $\begin{array}{c}\text { Mallet pré- } \\
\text { operatória }\end{array}$ & $\begin{array}{c}\text { Mallet pós- } \\
\text { operatória }\end{array}$ & $\begin{array}{c}\text { Aumento } \\
\text { pontuação }\end{array}$ \\
\hline & 10 & 13 & 3 \\
\hline 4 & 9 & 14 & 5 \\
\hline 5 & 9 & 12 & 3 \\
\hline 6 & 8 & 13 & 5 \\
\hline 8 & 8 & 13 & 5 \\
\hline 10 & 10 & 15 & 5 \\
\hline 11 & 8 & 14 & 6 \\
\hline & & & Média $=4,6$ \\
\hline
\end{tabular}

Grupo B: Paralisia completa

\begin{tabular}{|c|c|c|c|}
\hline Caso & $\begin{array}{c}\text { Mallet pré- } \\
\text { operatória }\end{array}$ & $\begin{array}{c}\text { Mallet pós- } \\
\text { operatória }\end{array}$ & $\begin{array}{c}\text { Aumento } \\
\text { pontuação }\end{array}$ \\
\hline 2 & 6 & 14 & 8 \\
\hline 3 & 8 & 8 & 0 \\
\hline 5 & 5 & 11 & 6 \\
\hline 7 & 8 & 13 & 5 \\
\hline & & & Média $=4,7$ \\
\hline
\end{tabular}

Os resultados pós-cirúrgicos são sobreponíveis no grupo A e B, verificando-se portanto, na nossa série, a eficácia da operação de L'Épiscopo modificada nas lesões completas do plexo braquial, facto este não defendido por muitos autores na literatura ${ }^{14}$. Na nossa experiência, parece-
TABELA V - REsultados SEgundo A

IDADE NA DATA DA CIRURGIA

Grupo I: Idade $\leq \mathbf{4}$ anos

\begin{tabular}{|c|c|c|c|c|}
\hline Caso & Idade & $\begin{array}{c}\text { Mallet pre- } \\
\text { operatória }\end{array}$ & $\begin{array}{c}\text { Mallet pos- } \\
\text { operatória }\end{array}$ & $\begin{array}{c}\text { Aumento } \\
\text { pontuação }\end{array}$ \\
\hline 2 & 3 & 6 & 14 & 8 \\
\hline 3 & 2 & 8 & 8 & 0 \\
\hline 4 & 3 & 9 & 14 & 5 \\
\hline 7 & 2 & 5 & 11 & 6 \\
\hline 8 & 3 & 8 & 13 & 5 \\
\hline 9 & 2 & 8 & 13 & 5 \\
\hline & & & & Média $=4,8$ \\
\hline
\end{tabular}

Grupo II: Idade $>\mathbf{4}$ anos

\begin{tabular}{|r|c|c|c|c|}
\hline Caso & Idade & $\begin{array}{c}\text { Mallet pre- } \\
\text { operatória }\end{array}$ & $\begin{array}{c}\text { Mallet pos- } \\
\text { operatória }\end{array}$ & $\begin{array}{c}\text { Aumento } \\
\text { pontuação }\end{array}$ \\
\hline 1 & 9 & 10 & 13 & 3 \\
\hline 5 & 5 & 9 & 12 & 3 \\
\hline 6 & 7 & 8 & 13 & 5 \\
\hline 10 & 6 & 10 & 15 & 5 \\
\hline 11 & 11 & 8 & 14 & 6 \\
\hline & & & & Media $=4,4$ \\
\hline
\end{tabular}

nos que a cirurgia paliativa do ombro em paralisias completas poderá estar indicada quando associada a cirurgias de recuperação da função do cotovelo, punho e/ou mão ${ }^{15}$.

Não se verificou relação entre a idade aquando da cirurgia e os resultados. Embora alguns autores ainda defendam que a cirurgia paliativa da POPB deverá ser realizada até aos 3 ou 4 $\operatorname{anos}^{14}$, na nossa experiência, idades superiores não contra-indicam a cirurgia, desde que sejam excluídas alterações significativas da articulação glenoumeral. Por outro lado, a criança com mais idade colabora melhor na reabilitação pós-cirúrgica, melhorando significativamente a função do ombro.

Contudo, é importante referir as limitações deste estudo: a amostra é reduzida e o seguimento curto. Além disso, a comparação entre os doentes com paralisia alta e completa é especulativa, uma vez que a avaliação final de Mallet no grupo de paralisia completa é o somatório de todas as cirurgias efectuadas ao membro superior. 


\section{CONCLUSÃO}

A técnica de L'Épiscopo modificada por Hoffer é eficaz no tratamento paliativo do ombro em POPB, pois a transferência do grande dorsal e grande redondo para a coifa dos rotadores permite estabilizar melhor a rotação externa e aumentar a eficácia do deltóide com ganho da abdução.
Apesar da nossa série ser limitada podemos admitir que este procedimento também tem bons resultados em paralisias completas, devendo no entanto ser associado a outras cirurgias de recuperação da função do cotovelo, punho e/ou mão. Além disso, também podemos considerar que a sua indicação poderá abranger crianças com idade mais avançada desde que sejam excluídas as alterações da articulação glenoumeral.

\section{BIBLIOGRAFIA}

1. Waters PM. Obstetrical braquial plexus injuries: evaluation and management. J Am Acad Orthop Surg, 1997; 5: 205-14.

2. Waters PM, Smith GR, JaramiIlo D. Glenohumeral deformity secondary to braquial plexus birth palsy. J Bone Joint Surg Am, 1998; 80: 668-77.

3. Dunkerton MC. Posterior dislocation of the shoulder associated with obstetric braquial plexus palsy. Br J Surg, 1989; 71: 764-6.

4. Greenwald AG, Schute PC, Shiveley JL. Braquial plexus birth palsy: a 10-year report on incidence and prognosis. $\mathrm{J} \mathrm{Pe}$ diatr Orthop, 1984; 4: 689-92.

5. Pearl ML, Edgerton BW. Glenoid deformity secondary to braquial plexus birth palsy. J Bone Joint Surg Am, 1998; 80: 659-67.

6. Sever JW. The results of a new operation for obstetrical paralysis. Am J Orthop Surg, 1918; 16: 248-57.
7. Gilbert A, Tassain JL. Reparation chirurgicale du brachial dans la paralysis obstetricale. Chirurgie, 1984; 110: 70-4.

8. Fairbank HAT. Birth palsy: subluxation of shoulder joint in infants and young children. Lancet, 1913; 1: 1217-23.

9. L'Episcopo JB. Tendon transplantation in obstetrical paralysis. Am J Surg, 1934; 25: 122-5.

10. Hoffer MM, Wickenden R, Roper B. Braquial plexus palsies: results of tendon transfers to the rotator cuff. J Bone Joint Surg Am, 1978; 60: 691-5.

11. Pearl MI, Edgerton BW, Kazimiroff PA, et al. Arthroscopic release and latissimus dorsi transfer for shoulder internal rotation contractures and glenohumeral deformity secondary to braquial plexus birth palsy. $\mathrm{J}$ Bone Joint Surg Am, 2006; 88: 564-74.
12. Gilbert A, Tassin J L. Microreconstruction of nerve injuries. En: Terzis JK (Ed). Obstetrical palsy: a clinical, pathologic, and surgical review. Philadelphia: WB Saunders; 1987; 52953.

13. Mallet J. Paralysie obstétricale du plexus brachial. II Thérapéutique. B. Traitement des séquelles. a) Primauté du traitement de l'epaule.- Méthode d'expression des résultats. Rev Chir Orthop, 1972; 58 (Suppl 1): 166-8.

14. Covey DC, Riordan DC, Milstead ME, et al. Modification of L'Episcopo procedure for braquial plexus birth palsies. $J$ Bone Joint Surg Br, 1992; 74: 897-901.

15. Raimondi P. Evaluation of results in obstetrical braquial plexus palsy. The Hand in proceedings of the International Meeting on Obstetrical Braquial Plexus Palsy, Heerlen, 1993. 